Med Klin Intensivmed Notfmed $2021 \cdot 116$ : 527-529

https://doi.org/10.1007/s00063-021-00832-1

Angenommen: 7. Mai 2021

Online publiziert: 11. Juni 2021

(c) Springer Medizin Verlag $\mathrm{GmbH}$, ein Teil von Springer Nature 2021

\author{
Uta Ochmann ${ }^{1} \cdot$ Sabine Wicker ${ }^{2} \cdot$ Guido Michels $^{3}$ \\ ${ }^{1}$ Institut und Poliklinik für Arbeits-, Sozial- und Umweltmedizin, Stabsstelle Betriebsärztlicher Dienst, LMU \\ Klinikum München, München, Deutschland \\ ${ }^{2}$ Betriebsärztlicher Dienst, Universitätsklinikum Frankfurt, Goethe-Universität Frankfurt am Main \\ Frankfurt am Main, Deutschland \\ ${ }^{3}$ Klinik für Akut- und Notfallmedizin, St.-Antonius-Hospital Eschweiler, Eschweiler, Deutschland
}

\section{Schwangere Mitarbeiterinnen im Gesundheitswesen: Schutz durch Impfen gegen SARS-CoV-2 und Tragen von FFP2-Masken}

fung in der Schwangerschaft empfohlen [2]. Dagegen kann Schwangeren mit Vorerkrankungen und einem daraus resultierenden hohen Risiko für eine schwere COVID-19-Erkrankung in Einzelfällen nach Nutzen-Risiko-Abwägung und nach ausführlicher Aufklärung eine Impfung angeboten werden.

Schwangere waren in den Zulassungsstudien für die Impfstoffe nicht inkludiert, jedoch haben Pfizer und Moderna offengelegt, dass 12 bzw. 6 Studienteilnehmerinnen zum Zeitpunkt der Impfung bereits unbekannt schwanger waren. Die Centers for Disease Control and Prevention (CDC) schätzt, dass aufgrund der Impfpriorisierung für Beschäftigte im Gesundheitswesen von diesen bereits ca. 300.000 Frauen unerkannt schwanger geimpft worden sein können. Somit sind künftig Daten zum Outcome dieser Schwangerschaften zu erwarten [3]. Eine jüngst publizierte Registerstudie (V-Safe) zu vorläufigen Ergebnissen bei über 30.000 trotz Schwangerschaft geimpften Frauen zeigte, dass impfbedingte Nebenwirkungen sich bis auf vermehrte lokale Schmerzen im Bereich der Impfung nicht von denen der Nichtschwangeren unterschieden [4]. Häufigkeit und Art von unerwünschten Schwangerschafts- und Neugeborenenereignissen waren vergleichbar mit COVID-19-Pandemie [4]. Schwangere weisen ein erhöhtes Risiko für schwere den vorbekannten Inzidenzen vor der
COVID-19-Verläufe mit einer erhöhten Früh- und Totgeburtsrate auf, sodass eine baldige Zulassung der Impfstoffe für diese Personengruppe wünschenswert ist.

Seit Ende 2020 stehen verschiedene Impfstoffe gegen SARS-CoV-2 zur Verfügung. Mitarbeiter im Gesundheitswesen, die in der direkten Patientenversorgung eingesetzt sind, gehören nach Impfverordnung der Priorisierungsgruppen 1 und 2 an. Innerhalb der letzten 4 Monate konnte somit einem Großteil der Mitarbeiter/-innen in der direkten Patientenversorgung eine Impfung angeboten werden.

Eine Datenanalyse aus Israel Ende Januar 2021 bei knapp 10.000 Beschäftigten im Sheba Medical Centre ergab vollständige Impfungen bei $66 \%$ der Beschäftigten. Im Vergleich zu nichtgeimpften Beschäftigten mit einer Rate von 7,4 Infektionen in 10.000 Personentage lag die Rate für vollständig geimpfte Beschäftigte bei 3,0 pro 10.000 Personentage. Die Risikoreduktion für vollständig geimpfte Beschäftigte wurde mit $60 \%$ berechnet. Wichtiger noch bei Betrachtung des Risikos für geimpfte Schwangere im Gesundheitswesen sind die Zahlen der Reduktion der symptomatischen Infektionsverläufe. Die Risikoreduktion wird diesbezüglich mit $75 \%$ angegeben. Bemerkenswert in dieser Studie ist auch die Angabe, dass $70 \%$ der 125 nachverfolgbaren Infektionsfälle (insgesamt 149 Fälle 
bei geimpften und nichtgeimpften Beschäftigten) im privaten Umwelt verursacht worden [5].

Die CDC hat gleichfalls Daten zu Beschäftigten im Gesundheitswesen im direkten Patientenkontakt aus dem Zeitraum Dezember 2020 bis März 2021 veröffentlicht. $62,8 \%$ waren vollständig geimpft. Die Inzidenzrate bei nichtgeimpften Beschäftigten betrug 13,8 pro 10.000 Personentage, für vollständig geimpfte Beschäftigte 1,9 pro 10.000 Personentage. Die Risikoreduktion wird mit $90 \%$ berechnet [6].

Nach den derzeitigen Ausführungen des Robert Koch-Instituts (RKI) gilt für vollständig geimpfte Personen mit engem Kontakt zu positiv getesteten Personen, dass sie nicht mehr in Quarantäne müssen. Hieraus ist abzuleiten, dass das Risiko einer Virusübertragung durch Geimpfte als sehr gering bewertet wird.

\section{Schutz durch Tragen von FFP2-Masken}

Zusätzlich zu der positiven Entwicklung der Immunisierung werden im Gesundheitswesen die Hygienevorschriften mit Tragen von chirurgischen Mund-NaseSchutz oder FFP2-Masken unverändert eingehalten. Auch Schwangere können nach Mutterschutzgesetz FFP2-Masken tragen, wenn diese nicht zu einer Belastung der Schwangeren führen.

Nach der Stellungnahme des Ausschusses für Arbeitsmedizin (AfaMed) vom 24.03.2021 [7] und den Ausführungen in der Deutschen Gesetzlichen Unfallversicherung(DGUV)-Regel 112190 [8] sollen Tragezeiten nach situationsbezogener Gefährdungsbeurteilung unter Einbeziehung arbeitsmedizinischer Expertise festgelegt werden. Dabei sind die mit der Maske durchzuführenden Tätigkeiten, die Arbeitsschwere und die Umgebungsbedingungen zu berücksichtigen. Da sich beim Tragen von FFP2Masken zum Zweck des Infektionsschutzes in nichtstaubiger normal temperierter Innenraumluft der ursprüngliche Atemwiderstand der FFP2-Maske nicht weiter erhöht, kann die Gefährdungsbeurteilung entsprechend angepasst werden. Hierzu wird auch auf die Ausführungen in den technischen Regeln für biologi- sche Arbeitsstoffe TRBA 250, Anhang 7 verwiesen [9].

Beschäftigte, die zum Infektionsschutz über fast die gesamte Arbeitszeit eine FFP2-Maske tragen, sollten auf jeden Fall die Möglichkeit haben, in einem Raum ohne erhöhte Infektionsgefährdung ihre Maske kurz abzunehmen, auch die regulären Arbeitszeitpausen müssen ohne Tragen von FFP2-Masken ermöglicht werden. Die trifft selbstverständlich auch für Schwangere zu, die nach Mutterschutzgesetz sowieso das Anrecht haben, jederzeit Pausen einlegen zu dürfen. Literatur zum Thema Tragen von FFP2-Masken in der Schwangerschaft ist rar. Ein kleiner systematischer Review aus dem Jahr 2020 führt 3 Studien auf mit Tragezeiten von maximal $1 \mathrm{~h}$ anteilig mit körperlichen Belastung über $20 \mathrm{~min}$ mit $50 \mathrm{~W}$ respektive 3 MET (metabolisches Äquivalent). Hierbei konnten keine signifikanten Änderungen der kardiopulmonalen Belastungsparameter gezeigt werden. Studien zu längerem Tragen wurden nicht gefunden [10].

\section{Fazit für die Praxis}

Vor dem Hintergrund der obigen Daten muss aus Sicht der Autoren die Gefährdungsbeurteilung für Schwangere im Gesundheitswesen aktualisiert werden. Es ist nicht nachvollziehbar, warum die für die Umsetzung des Mutterschutzgesetzes zuständigen Aufsichtsbehörden einiger Bundesländer weiterhin pauschale Beschäftigungsverbote für Schwangere aussprechen, insbesondere, wenn diese in stationären Bereichen tätig sind. Nach Mutterschutzgesetz ist eine Benachteiligung von Schwangeren zu vermeiden, eine individuelle Beurteilung der Gefährdung ist vorgeschrieben. Durch die Maßnahmen Impfung und Tragen von FFP2-Masken sind auch schwangere Mitarbeiterinnen im Gesundheitswesen hinreichend geschützt. In Abhängigkeit von einer individuellen Gefährdungsbeurteilung ist unter Einhaltung der Infektionsschutzmaßnahmen auch eine Beschäftigung im direkten Patientenkontakt möglich.

\section{Korrespondenzadresse}

\section{Dr. med. Uta Ochmann}

Institut und Poliklinik für Arbeits-, Sozial- und Umweltmedizin, Stabsstelle Betriebsärztlicher Dienst, LMU Klinikum München

Ziemssenstraße 1, 80336 München,

Deutschland

uta.ochmann@med.uni-muenchen.de

\section{Einhaltung ethischer Richtlinien}

Interessenkonflikt. S. Wicker ist stellvertretende Vorsitzende der Ständigen Impfkommission (STIKO). G. Michels ist Sprecher der Arbeitsgruppe Kardiopulmonale Reanimation (AG42), stellvertretender Sprecher des Cluster A: Kardiovaskuläre Akut- und Intensivmedizin und Sprecher des Arbeitskreises Mechanische Kreislaufunterstützung (AK-MCS) der Arbeitsgruppe Interventionelle Kardiologie der Deutschen Gesellschaft für Kardiologie e. V. (DGK); er erhielt gelegentlich Honorare für Vortragstätigkeiten von Pfizer, Novartis, Servier, Zoll, Getinge und Orion Pharma. Die Interessenkonflikte stehen in keinem Zusammenhang mit dem vorliegenden Konsensuspapier. U. Ochmann ist Vorsitzende des Ausschusses für Mutterschutz beim BMFSFJ, sie gibt an, dass keine Interessenkonflikte im Zusammenhang mit dem Inhalt des Beitrags bestehen.

Für diesen Beitrag wurden von den Autoren keine Studien an Menschen oder Tieren durchgeführt. Für die aufgeführten Studien gelten die jeweils dort angegebenen ethischen Richtlinien.

\section{Literatur}

1. https://www.aerzteblatt.de/nachrichten/ 123219/Ein-Drittel-der-Intensivpflegendenwill-Arbeitsstelle-aufgeben. Zugegriffen: 23. Apr. 2021

2. Beschluss der STIKO zur 4. Aktualisierung der COVID-19-Impfempfehlung. https://www.rki.de/DE/Content/Infekt/ EpidBull/Archiv/2021/16/Art_01.html; jsessionid=7BAE0BF201886F481B4690CB791A4 E66.internet122.Zugegriffen:24. Apr. 2021

3. Rasmussen SA, Kelley CF, Horton JP, Jamieson DJ (2021) Coronavirus disease 2019 (COVID-19) vaccines and pregnancy. Obstet Gynecol 137:408-414

4. Shimabukuro TT, Kim SY, Myers TR et al (2021) Preliminary findings of mRNA Covid-19 vaccine safety in pregnant persons. N Engl J Med. https:// doi.org/10.1056/NEJMoa2104983

5. Amit S, Regev-Yochay G, Afek A, Kreiss Y, Leshem E (2021) Early rate reductions of SARS-CoV-2 infection and COVID-19 in BNT162b2 vaccine recipients. Lancet 397:875-877

6. Thompson MG, Burgess JL, Naleway AL et al (2021) Interim estimates of vaccine effectiveness of BNT162b2 and mRNA-1273, COVID-19 vaccines in preventing SARS-CoV-2 infection among health care personnel, first responders, and other essential and frontline workers-Eight U.S. Locations, December 2020-March 2021. MMWR Morb Mortal Wkly Rep 70(13):495-500 
7. https://www.baua.de/DE/Aufgaben/ Geschaeftsfuehrung-von-Ausschuessen/ AfAMed/pdf/Stellungnahme-Tragezeit-FFP2Masken.pdf?_blob=publicationFile\&v=3.

Zugegriffen:24. Apr. 2021

8. https://publikationen.dguv.de/widgets/pdf/ download/article/1011.Zugegriffen:24. Apr. 2021

9. https://www.baua.de/DE/Angebote/Rechtstexteund-Technische-Regeln/Regelwerk/TRBA/pdf/ TRBA-250.pdf? blob=publicationFile\& $v=4$. Zugegriffen:24.Apr. 2021

10. Roeckner JT, Krstić N, Sipe BH, Običan SG (2020) N95 filtering facepiece respirator use during pregnancy: a systematic review. Am J Perinatol 37:995-1001

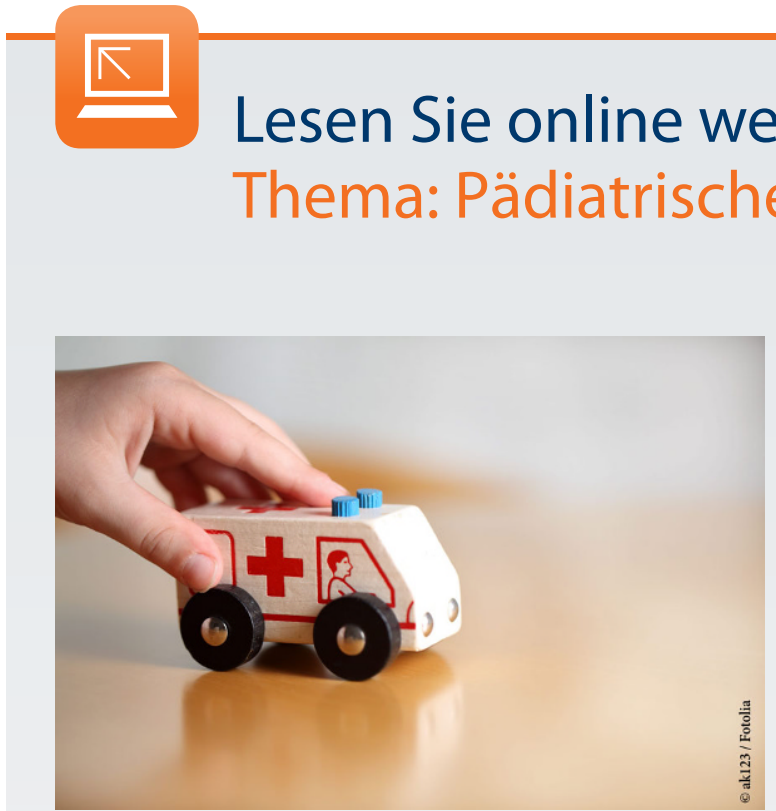

Lesen Sie auf SpringerMedizin.de weiter: Auf der Themenseite Pädiatrische Notfall- und Intensivmedizin finden Sie Handlungsempfehlungen für den Kindernotfall, Kasuistiken aus der pädiatrischen Intensivmedizin, Aktuelles zu Brandwunden, Ertrinken oder Reanimation, einiges davon aus Ihrer Zeitschrift und noch mehr aus dem kompletten Portfolio von Springer Medizin.

Über diesen QR-Code kommen Sie direkt auf die Themenseite „Pädiatrische Notfall- und Intensivmedizin“. Einfach mit der Handykamera scannen und los gehts es!

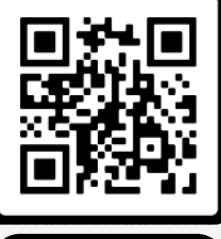

SCAN ME
Sind Sie schon bei SpringerMedizin.de registriert?

Als registrierter Kunde haben Sie bereits Zugriff auf viele Inhalte der Seite. Die Registrierung ist nötig, da es sich bei SpringerMedizin.de um ein Fachportal für Mitarbeiter aus dem Gesundheitswesen handelt.

- Registrieren Sie sich einmalig auf

www.springermedizin.de/register.

- Geben Sie dafür Ihre Einheitliche Fortbildungsnummer (EFN) an, hinterlegen Sie einen entsprechenden Berufsnachweis (Notfallsanitäter, Pfleger) oder schicken Sie einen Studiennachweis mit Angabe des Studiengangs. Wenn Sie zusätzlich noch ein Abonnement haben (Zeitschrift oder e.Med), dann erweitert sich Ihre Berechtigung entsprechend.

\section{Schalten Sie Ihre Abonnements frei!}

- Geben Sie bei der Registierung die Lieferadresse Ihrer Zeitschrift an, dadurch wird Ihr Abo-Zugang automatisch auf SpringerMedizin.de freigeschaltet.

- Unter "Mein Profil“ können Sie neue Abonnements oder Berechtigungen über eventuelle Gesellschaftsmitgliedschaften hinzufügen und Ihre Daten oder das Passwort ändern.

- Tipp: Sollten die Angaben Ihres Online-Accounts nicht eindeutig mit den Angaben Ihres Zeitschriften-Abonnements übereinstimmen, kann die Zuordnung nicht sicher erfolgen.

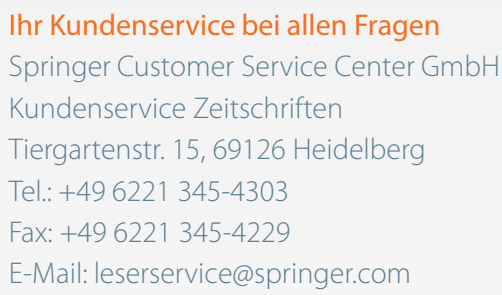

